\title{
Lumen
}

Selected Proceedings from the Canadian Society for Eighteenth-Century Studies

\section{“ Le soc s'est ennobli sous les mains d'un bon roi » - lumières, nature et bonheur à la veille de la Révolution ou réaction nobiliaire? Le cas de Lezay-Marnésia}

\section{Roland Bonnel}

Volume 12, 1993

URI : https://id.erudit.org/iderudit/1012586ar

DOI : https://doi.org/10.7202/1012586ar

Aller au sommaire du numéro

Éditeur(s)

Canadian Society for Eighteenth-Century Studies / Société canadienne d'étude du dix-huitième siècle

ISSN

1209-3696 (imprimé)

1927-8284 (numérique)

Découvrir la revue

Citer cet article

Bonnel, R. (1993). « Le soc s'est ennobli sous les mains d'un bon roi » - lumières, nature et bonheur à la veille de la Révolution ou réaction nobiliaire? Le cas de Lezay-Marnésia. Lumen, 12, 139-147. https://doi.org/10.7202/1012586ar

Copyright (c) Canadian Society for Eighteenth-Century Studies / Sociéte canadienne d'étude du dix-huitième siècle, 1993
Ce document est protégé par la loi sur le droit d'auteur. L'utilisation des services d'Érudit (y compris la reproduction) est assujettie à sa politique d'utilisation que vous pouvez consulter en ligne.

https://apropos.erudit.org/fr/usagers/politique-dutilisation/ 


\section{3. 'Le soc s'est ennobli sous les mains d'un bon roi' - lumières, nature et bonheur à la veille de la Révolution ou réaction nobiliaire? Le cas de Lezay-Marnésia}

Cette citation est du marquis Claude-François-Adrien de LezayMarnésia ${ }^{1}$ dont l'oeuvre contribue à clarifier l'ambiguïté de la pensée de la noblesse éclairée dans les années 1780 et à mieux comprendre la crise d'identité du second ordre. Si Lezay-Marnésia était appelé à son époque ' 1 'Aigle ou le Cygne patriotique du Mont Jura,' ${ }^{2}$ il passe aujourd'hui inaperçu, et s'il est mentionné, c'est toujours brièvement: à propos de la poésie descriptive, de la préparation des Etats Généraux ou de la francmaçonnerie spiritualiste. Cependant c'est sa conformité aux idées du temps, aux modes de son milieu et les hésitations de sa pensée qui font l'intérêt du personnage, outre le fait qu' 'il a laissé le souvenir d'un homme de bien, profondément désintéressé qui voulut le progrès pur de tout excès. ${ }^{3}$

Lorsqu'en 1759 il publie un article dans le Journal encyclopédique, qui sera repris dans l'Encyclopédie, c'est pour compatir au sort de la paysannerie. Le même intérêt pour la condition des campagnes transparaît dans le conte moral $L^{\prime}$ 'Heureuse famille. ${ }^{5} \mathrm{Si}$ Grimm juge ce conte 'fort insipide, ${ }^{\prime}$ il n'en reste pas moins intéressant car il révèle déjà en Lezay-Marnésia un idéaliste sincèrement généreux dont l'amour de la nature semble s'exprimer surtout de façon littéraire. Son discours de réception à l'Académie de Nancy en 1767 confirme ce jugement. Fréron cite des extraits de ce discours dans l'Année Littéraire: 'l'étude de l'homme champêtre est pour l'homme de lettres ce que l'étude du nu est pour le peintre. S'il n'acquiert pas l'intelligence parfaite du caractère original, jamais il ne mettra de vérité dans ses portraits' (23 févier 1768; ii, 26). En 1787 Lezay-Marnésia s'avoue disciple de Delille et de Saint-Lambert et publie son Essai sur la nature champêtre qui fera dire à La Harpe que l'auteur 'a plus d'amour pour la campagne que de talent pour la versification,' tout en lui accordant 'le naturel que donne un sentiment vrai' et la sincérité, ${ }^{8}$ 
jugement que partagent la Correspondance littéraire (Grimm; août 1787, xv, 115-16) et l'Année Littéraire (3 août 1787, vi, 112-20).

Dans l'intervalle de ces publications, son amour des campagnes, si littéraire fût-il, n'était pas resté théorique: en 1769 il avait démissionné de l'armée pour s'installer dans ses domaines. Il y avait créé des jardins, s'était consacré à l'agriculture et à l'amélioration de la condition paysanne en finançant des hôpitaux, des écoles, des centres communautaires. La bienfaisance était alors la mode, mais au coeur d'une FrancheComté encore féodale il devait en dépasser les limites en abolissant dans ses terres la mainmorte et la corvée et en réclamant l'égalité devant l'impôt. En 1788 il finance le Mémoire du peuple français de Cerutti qui dénonce les privilèges, et en 1789 on le compte dans les rangs de la minorité de la noblesse. L'abbé Lambert a ainsi résumé la carrière politique de Lezay-Marnésia: 'Constituant, il avait été un des quarantecinq qui s'étaient réunis au tiers avant leur ordre, et, pour le fond de son système il était Anglais. ${ }^{9}$

C'est en 1785 que paraît la première édition du Bonheur dans les campagnes. ${ }^{10} \mathrm{Il}$ s'agit d'une oeuvre à la fois morale, littéraire et politique; chaque thème a un lexique spécifique, si bien que, autant que les idées, la façon dont elles sont exprimées à travers différents codes lexicaux et stylistiques révèle la pensée de l'auteur. Le propos de l'oeuvre est clairement formulé dans l'introduction: il s'agit de chercher des remèdes aux maux qui affligent les campagnes. Pour décrire la campagne, le lexique est celui de la maladie ${ }^{11}$ car 'le tableau le plus pathétique de la misère excite l'émotion' (2); pour décrire la ville, il est moral et négatif. ${ }^{12}$ Le constat se fonde ainsi sur des associations qualitatives d'ordre affectif et social. La phrase de Voltaire placée en exergue - 'c'est la cour qu'on doit fuir; c'est aux champs qu'il faut vivre' - est ainsi expliquée par Lezay-Marnésia: 'la cause du peuple est si belle à défendre, il est si doux de s'occuper des moyens de le rendre plus heureux, que l'âme sensible se plaît toujours à les chercher, espère qu'elle les trouvera, et se flatte même, dans son ivresse, qu'ils seront adoptés' (168). C'est l'appel à la noblesse de retourner dans ses châteaux, appel déjà lancé par ses amis Saint-Lambert ${ }^{13}$ et Philippon de la Madeleine ${ }^{14}$ et par Mirabeau, ${ }^{15}$ pour ne citer que des exemples bien connus de Lezay-Marnésia. L'agronomie est à la mode et Lezay-Marnésia suit la mode; il ne doute pas que la richesse repose sur la propriété foncière et que le devoir du riche soit d'oeuvrer au bonheur du pauvre. Mais cette injonction tient de la prescription médicale: la campagne a une valeur thérapeutique et l'appel à la bienfaisance ne dépasse pas l'individu, l'émotion induite chez le bienfaiteur étant tout aussi importante, sinon plus, que la condition paysanne. 
Néanmoins, l'oeuvre de Lezay-Marnésia est plus qu'un traité du bonheur; elle s'inscrit dans la tradition nobiliaire, agraire, patriarcale et 'socialisante'; elle est à la fois nostalgie de l'Age d'Or et espérance de régénération. Critiquant l'ordre établi, il accuse Richelieu et l'absolutisme d'être à l'origine de la misère des campagnes et propose des réformes. Comme l'indique l'Année littéraire (décembre 1789, viii, 330$40){ }_{1}^{16}$ que ces idées aient été répandues dans les milieux éclairés n'enlève rien à leur portée. Il s'agit d'abord de mettre fin aux abus en moralisant la fonction publique et en utilisant le critère d'utilité sociale pour évaluer le rôle du clergé. Il faut ensuite rétablir l'équité en répartissant plus justement l'impôt et en développant l'instruction dans les campagnes à la mesure des besoins. Enfin il est question de mettre fin à l'arbitraire en créant des états provinciaux, intermédiaires entre le roi et le peuple. $C^{\prime}$ est redonner là à la noblesse un rôle politique qui ne sera plus fondé sur des valeurs aristocratiques mais sur la valeur bourgeoise du mérite. Par exemple, Lezay-Marnésia suggère de créer un nouvel ordre de chevalerie, l'Ordre patriotique. Il serait décerné aux personnes sensibles aux problèmes ruraux. Les 'bienfaiteurs des campagnes' recevraient ainsi une médaille figurant une charrue entourée d'une couronne d'or qui imiterait des épis de blé et dont la devise serait: 'le soc s'est ennobli sous les mains d'un bon roi' (71).

Pourtant il n'est nulle part question d'abolir les privilèges ou l'impôt seigneurial comme chez Clicquot de Blervache dont la pensée est pourtant voisine. ${ }^{17}$ Lezay-Marnésia s'oppose même ouvertement à la division des terres. La prospérité publique repose à ses yeux sur l'intérêt privé, et le niveau réel de la vie sociale est celui de la communauté villageoise rassemblée autour du bienfaiteur. S'il diagnostique l'abus des privilèges comme la source de la pauvreté des campagnes, l'ordre aristocratique n'est pas menacé; au contraire, il s'en trouve renforcé car l'abus des privilèges n'est pas son fait mais celui d'une noblesse de cour qui s'est éloignée de ses devoirs. Le bon noble est celui qui met fin aux abus des fonctionnaires corrompus. Alors que le 'citoyen' n'existe que dans l'acte d'obéissance, le noble, défini comme l'ami du Roi, a des fonctions bien établies: il est modèle pour la nation, protecteur des pauvres et intermédiaire entre le roi et le peuple. L'état de nature est remplacé par un monde où la nature perd son innocence si les nobles en sont absents. C'était transposer en traité l'idéal de la poésie descriptive: les bergers d'Arcadie ont été remplacés par des gentilhommes bien intentionnés. C'était aussi faire profession d'académisme et prendre à son compte un lieu commun des Lumières: guider le peuple, lui offrir un double modèle.-D'une part, l'élite est éducatrice et conseillère: elle offre un modèle de conduite. Comme l'écrit Fresnais de Beaumont, lui aussi noble et agronome, il faut aux 
côtés des laboureurs 'des gens aussi honnêtes, aussi actifs qu'eux, mais plus éclairés. ${ }^{18} \mathrm{D}$ 'autre part, la campagne, derrière laquelle se profile l'Age d'Or, n'est pas seulement prétexte esthétique ou sentimental, mais aussi modèle de société. La culture s'en trouve ennoblie et l'agriculture devient école de vertu, comme on peut le lire dans le Journal oeconomique: 'L'agriculture ... est de toutes les occupations de l'homme la plus noble et la plus avantageuse ... [elle éloigne l'agriculteur] d'un grand nombre de vices, leur rend familière la pratique de beaucoup de vertus' (janvier 1751, 6-7).

L'édition de 1788 du Bonheur dans les campagnes marque encore mieux le rôle de la noblesse. Les additions portent sur le roi, l'administration et les ordres. La pensée de Lezay-Marnésia révèle ici la symbiose entre une pensée sociale communautaire et une pensée politique traditionaliste. $D^{\prime}$ une part, il loue l'administration provinciale et salue la naissance $d$ 'un esprit national issu de la réunion des ordres; $d$ 'autre part, il dépeint le roi comme le père du peuple et le trône comme le dispensateur des Lumières, de l'assistance et de la protection. Dans les chapitres consacrés aux ordres, il recommande qu'un quota limitant les annoblissements soit institué. Enfin, il invite à la division du tiers état entre le tiers proprement dit, c'est-à-dire la bourgeoisie, et le peuple. Chaque catégorie sociale aura une fonction bien définie: le peuple obéira, le tiers état sera responsable du commerce, et la noblesse sera 'l'ornement, le soutien du trône, le boulevard de l'empire' (88). La crise d'identité de la noblesse se trouve ainsi résolue dans la relation père-fils, dans une dimension pédagogique et dans une conception organique de la société servant de justification à la hiérarchie des ordres.

Lezay-Marnésia était académicien (Besançon, Nancy et Lyon), poète et franc-maçon (Amis Réunis, Neuf Soeurs, Sincérité et Parfaite Union). Il partageait la pensée de milieux attachés à l'idée qu'une révolution prise au sens de restauration d'un modèle antique - était nécessaire. Malheureusement, il ne la définit pas clairement sinon dans des envolées oratoires. A ses yeux, elle ne pourra advenir que par la vertu, terminologie qui rappelle celle des Lumières mais dont le sens est différent. Influencé par le spiritualisme des Chevaliers Bienfaisants de la Cité Sainte, Lezay-Marnésia en fait une qualité spirituelle. La société issue de cette révolution sera celle de l'Age d'Or où les liens sont ceux de l'effusion sentimentale. Le Bonheur dans les campagnes culmine sur ce tableau de la famille seigneuriale vertueuse:

Une épouse pénétrée d'estime et de tendresse pour son époux, un mari tendre et heureux des vertus de sa femme, ... une famille toujours occupée d'objets utiles, où l'ordre fait régner l'abondance, que la gaieté n'abandonne jamais et 
que la piété, la bienfaisance animent toujours! De ce château que le bonheur habite, il [le seigneur] se répand sur les villages qui l'environnent, ... il en a banni les vices; il les a remplacés par l'activité, l'industrie et la sagesse. Juge, médecin, et père, par l'autorité de l'amour et du respect, il arrange les procès et visite les malades. ... Tout est en mouvement autour de lui ... et ses douces soirées se passent à recevoir les caresses de sa femme et de ses enfants, et à former de nouveaux projets pour le lendemain. (éd. 1785, 56-57)

Les moyens à mettre en oeuvre pour réaliser ce rêve sont présentés dans le projet de communauté que Lezay-Marnésia détaille dans ses Lettres de l'Ohio. ${ }^{19}$ Le modèle est celui du Salente de Fénelon, du Clarens de Rousseau, des communautés des frères moraves et des communautés anabaptistes de la région de Montbéliard. Si les premiers colons s'embarquent au printemps 1790, l'idée date d'avant la convocation des États Généraux. Lezay-Marnésia avait acheté avec quelques amis, parmi lesquels on compte Duval d'Eprémesnil, Lally-Tollendal, Mounier, Malouet, quelque 24000 acres en Amérique par l'intermédiaire de la Compagnie du Scioto. Ces fondateurs étaient des philanthropes rêveurs et de grands admirateurs de Bernardin de Saint-Pierre. Mais en 1790 il s'agissait aussi de chercher outre-atlantique 'la paix, la sûreté, la vraie liberté, dans une contrée innocente et tranquille, ${ }^{20}$ ce que ne manquèrent pas d'interprêter à leur façon les journaux de l'époque. Pour la Chronique de Paris, par exemple, le projet de Duval d'Eprémesnil a pour but d'établir aux Etats-Unis 'le régime féodal, le magnétisme, le mesmérisme, le théosophisme et le parlementarisme. ${ }^{21}$ Si pour certains le Scioto représente un refuge et une occasion de spéculation foncière, pour Lezay-Marnésia la spéculation est d'ordre moral, philosophique et religieux. Cette fondation est dans la logique de sa pensée. Rien, non pas même l'expression de son désenchantement sur ce qui se passe en France, ne permet d'affirmer qu'il se considère comme un 'émigré,' du moins au sens politique. Il ne conçoit pas son exil comme temporaire et refusera d'ailleurs toujours la solution politique de la contre-révolution. Les lettres qu'il écrit à sa femme en 1789 et $1790^{22}$ prouvent qu'il envisage une fondation définitive qui, si elle se trouve hâtée par la situation politique, reste vouée à la recherche des vertus primitives. Cette communauté, qu'il envisage de nommer Gallipolis, est celle d'un lecteur de la Nouvelle Héloïse et d'un agronome. Le fait qu'elle soit appelée 'colonie' et non 'société' révèle les limites de la conscience politique du fondateur. En effet, la notion de souveraineté populaire n'apparaît nulle part dans les Lettres de l'Ohio; la communauté est plus importante que la société civile dans laquelle elle s'inscrit. 
Elle se présente en effet comme une fondation privée soumise aux lois de l'état de Pennsylvanie, d'une utopie - dystopie dirait Delaporte ${ }^{23}$ dont la fin est de restaurer l'Age d'Or. Vu la conjoncture, ce microcosme prend une valeur sotériologique: la capitale, Saint-Pierre, nommée d'après Bernardin de Saint-Pierre, doit conserver les usages français et préserver les lettres, les sciences et les arts. Les noms des rues sont prévus: Fénelon, Pascal, Buffon, Rousseau, Racine, Corneille, Vincent de Paul, Sully, Necker, Montesquieu, et bien d'autres modernes. LezayMarnésia pense ainsi réaliser l'espoir de bien des membres de la République des Lettres: l'expérimentation in vivo d'une utopie. C'est pourquoi la colonie, sorte de laboratoire, doit être isolée, comme il l'explique dès le 9 novembre 1789 dans une lettre à sa femme: 'nous serons comme Idoménée quand il édifiait Salente, nous bâtirons des murs, nous ferons nos lois. ${ }^{24}$ Le but en est de protéger la colonie des vices du monde extérieur pour que ses habitants puissent y 'couler des jours paisibles et purs dans les douceurs de la vie patriarchale, dans les charmes de la vie fraternelle' et 'se livrer au bonheur d'adorer Dieu, de pratiquer les vertus que la religion enseigne, commande et récompense. ${ }^{25}$ Le projet baigne dans la religiosité. Les rapports entre les habitants sont ceux de l'amitié, de la tendresse et de la fraternité, et s'expriment dans des banquets au cours desquels se pratique une 'chaîne d'union' - terme emprunté au lexique maçonnique. Les habitants vivent dans l'état de nature 'qui n'est pas ... la vie sauvage, qui n'est qu'une dégénération, mais l'état où l'homme est sorti de la main du Créateur et où il a été instruit par Dieu même. ${ }^{26}$ Comme les colons seront peu nombreux, l'égalité sera possible; mais n'est envisagée que l'égalité naturelle qui ne contredit pas la répartition de la population en grades eu égard à l'utilité sociale et au mérite.

Le monarque et la noblesse ont laissé la place aux propriétaires terriens. A côté de la religion et de la raison, piliers de la colonie, le principe fondateur est la terre, comme à Salente. La colonie est une copropriété; l'administration, une gestion de 'propriétaires associés'; l'unité de base, la famille au sens antique du terme: la cellule familiale, ses domestiques, ses cultivateurs, ses artisans. Le gouvernement est de nature aristocratique: les familles - Lezay-Marnésia limite leur nombre à cent, encore qu'il estime le chiffre de cinquante préférable - nomment douze administrateurs renouvelables par quart tous les ans et ayant à charge les affaires de la colonie. Lezay-Marnésia reconnaît qu'en dépit de l'amour de la démocratie on ne peut mettre côte à côte propriétaires et fermiers - ou artisans - en raison des différences de ressources et d'éducation. De même, aucun fermier, artisan ou ouvrier ne peut s'installer dans la ville, coeur de la colonie. Il en donne les raisons: 'je pense que le calme le plus doux doit toujours y régner. ${ }^{27}$ Tout au plus les plus 
laborieux et les plus sages parmi eux pourront-ils avoir dans les faubourgs la jouissance de maisons propres qui resteront acquises à la société des propriétaires. Les relations propriétaires-salariés sont définies sans équivoque: les propriétaires devront à leurs salariés 'des soins, de la protection, le prix abondant de leur travail, de la douceur, de l'indulgence et de la bonté. Rien au delà. ${ }^{28}$

Il faut faire le voyage de Saint-Pierre pour saisir le sens de cette communauté. ${ }^{29}$ Près de l'enceinte de la ville: l'hospice de charité pour les malades, confié aux soeurs de Saint Vincent de Paul, et les manufactures. $S^{\prime}$ il faut veiller aux avantages du commerce, la conservation des moeurs n'en est pas moins impérative: les manufactures seront donc hors-les-murs. Puis, les faubourgs, avec les maisons des ouvriers, artisans et laboureurs qui l'auront mérité. A l'intérieur de l'enceinte, des 'maisons sans faste, sans décoration, mais riantes, agréables et même élégantes [qui] toutes devront avoir des jardins [car] autant il est sage de ne pas porter dans les champs le tableau des villes, autant il est raisonnable de retracer dans les villes les images des champs. ${ }^{30}$ Dans ces maisons vivront épisodiquement les familles de propriétaires. Le centre de la ville possèdera l'architecture la plus significative de la colonie. Il comportera une place en forme de croissant de lune, au symbolisme sans doute à rechercher dans la symbolique maçonnique. Au centre de la partie concave se dressera une colonne; couverte d'inscriptions, elle représente dans la maçonnerie l'ensemble des sciences et des arts; ici, elle portera le nom des fondateurs. Au centre de la partie convexe se trouvera le temple au fronton duquel seront gravés les mots 'Doctrine et Vérité' et dont les deux premiers pilastres supporteront les Tables de la Loi. ${ }^{31}$ Aux extrémités du croissant: d'un côté le collège universitaire, placé sous la direction de l'évêque et des magistrats, de l'autre côté une maison d'éducation pour les filles. Enfin, à mi-chemin entre le temple et le collège: le Palais de Justice, et entre le temple et l'école des filles: le' centre communautaire destiné aux assemblées, aux marchés et à des repas où seront conviés fermiers et artisans qui ainsi 'feront mieux que de se donner des égaux. Ils se donneront de tendres et de constants amis. $^{32}$

Le projet de Gallipolis, basé sur la conviction que la propriété est le véhicule le plus puissant pour améliorer l'agriculture, représentait pour Lezay-Marnésia la restauration de l'Age d'Or, la solution à la disproportion des richesses et au problème du luxe puisque tous seraient assez riches pour vivre dans l'abondance sans l'être suffisamment pour être tentés par le faste. Les profits, consacrés aux dépenses publiques, devaient ainsi permettre de réaliser le but de la société: 'le plus grand bonheur dans l'égalité et dans les sentiments d'une fraternité tendre. ${ }^{33}$ Beaunier a décrit Marnésia comme un 'poète, philanthrope, voyageur et 
toqué. ${ }^{34} \mathrm{~S}^{\prime} \mathrm{il}$ y a du vrai dans cette remarque, il faut ajouter aussi que Lezay-Marnésia n'a rien de ces 'faussaires des Lumières,' décrits par Chaussinand-Nogaret, ${ }^{35}$ qui avaient joué aux apprentis sorciers avant de se réveiller horrifiés par les forces qu'ils avaient libérées. L'oeuvre de Lezay-Marnésia permet, grâce à son honnêteté, de mesurer les limites de la politique des Lumières. Elle aide à mieux comprendre l'échec de la noblesse éclairée dans les tentatives qu'elle a faites pour concilier la critique du système et la réforme des abus avec les principes d'une révolution populaire qui n'allait pas être une simple restauration.

\section{ROLAND BONNEL}

Dalhousie University

\section{Notes}

1 Pour les données biographiques, on se reportera aux notes de mon article 'Le traitement de la nature dans Le Bonheur dans les campagnes,' in Roland Bonnel, ed., Facets of the Eighteenth-Century: Descriptive, Social and Normative Discourse (Toronto: Captus, 1991), 109-118.

2 Joseph-Antoine Cerutti, Epître en vers irréguliers sur les Jardins, l'Agriculture, les Assemblées provinciales, les ministres, les parlements, la Tolérance, etc. (Paris, 1790), in 'Avis de l'éditeur.'

3 'Eloge du marquis de Lezay-Marnésia' par $\mathrm{H}$. Thivier à l'Académie de Besançon, séance publique du 31 janvier 1878 (Mémoires de l'Académie des Sciences, Belles-Lettres et Arts de Besançon [Besançon, 1879], 18).

4 L'article parut sans nom d'auteur dans le numéro du 15 juillet 1759 . Il fut reproduit sous le titre 'Maraudeur' dans l'article 'Voleur,' Encyclopédie ou Dictionnaire raisonné des Sciences, des Arts et des Métiers (Neufchastel, 1765), xvii, 450-451.

5 L'Heureuse famille, conte moral (Genève et Nancy, 1766).

6 Frédéric-Melchior Grimm et al., Correspondance littéraire, philosophique et critique, ed. Maurice Tourneux (Paris, 1877-1882), 15 septembre 1766, vii, 127.

7 Essai sur la nature champêtre, en vers, avec des notes (Paris, 1787).

8 La Harpe, Correspondance littéraire... (Paris, 1801-1807), lettre ccxxxvii, volume v, 91.

9 Mémoires de famille de l'abbé Lambert sur la révolution et l'émigration 1791-1799, publiés par G. de Beauséjour (Paris, 1894), 88.

10 Le Bonheur dans les campagnes (Neufchatel et Paris, 1785).

11 par exemple: 'remèdes,' 'maux,' 'douleurs,' 'mal,' 'souffrir,' 'languir,' 'gémir.'

12 Par exemple: 'cupidité,' 'paresse,' 'vices,' 'oisiveté,' 'flatterie,' 's'étourdir,' 'mollesse,' 'frivolité.' 
13 Les Saisons, poème (Amsterdam, 1769).

14 Dans son discours lu devant l'Académie de Besançon, séances des 27 septembre et 6 octobre 1782. Foñ de l'Académie de Besançon, ms 9, 381.

15 L'Ami des Hommes, ou Traité de la population (s.l., 1758-1760).

16 A l'occasion de l'édition de 1788 du Bonheur dans les campagnes.

17 Simon Clicquot de Blervache, Essai sur les moyens d'améliorer en France la condition des laboureurs, des journaliers, des hommes de peine vivant dans les campagnes et celle de leurs femmes et de leurs enfants, par un Savoyard (Chambéry, 1789), 52-53.

18 Fresnais de Beaumont, La noblesse cultivatrice (Paris, 1778), 5.

19 Lettres écrites des rives de l'Ohio (Fort Pitt et Paris, an ix).

20 Extrait de la lettre du 9 août 1790 du marquis de Lezay-Marnésia à d'Eprémesnil, citée par Henri Carré, 'Les Emigrés français en Amérique, 1789-1793,' La Revue de Paris, 15 mai 1898, 316.

21 Cité par Carré 320.

22 Ces lettres se trouvent dans des archives privées. Des extraits en ont été publiés dans Elisabeth Bourget-Besnier, Une famille française sous la Révolution et l'Empire. La famille de Lezay-Marnésia (Paris, 1985).

23 André Delaporte, L'idée d'égalité en France au XVIIIe siècle (Paris, 1987), 188.

24 Cité par Bourget-Besnier, 26.

25 Lettres de l'Ohio, 14-15.

26 Ibid., 60.

27 Ibid., 88.

28 Ibid., 74.

29 Je tiens à remercier ici Marie-France Silver et Pierre Gobin qui, lors de la discussion qui a suivi ma communication, ont attiré mon attention sur la ressemblance étonnante entre l'architecture de Saint Pierre/Gallipolis et les salines d'Arc-et-Senans. Il est certain que Lezay-Marnésia connaissait les constructions de Ledoux. Sa propriété de Saint-Julien était proche des salines et la route qu'il empruntait pour aller dans les propriétés de sa femme y passait. Cependant, alors que les projets de Ledoux pour la ville de Chaux intègrent habitat, jardins et industries, le projet de Lezay-Marnésia instaure une séparation entre habitat et jardins d'une part, industries de l'autre. L'utopie marnésienne reste soumise aux limites de la pensée des Lumières en matière de réformes.

30 Lettres de l'Ohio., 70-72.

31 Cette symbolique maçonnique n'étonnera pas chez Lezay-Marnésia qui avait occupé des grades élevés et avait même reçu tous ses domestiques franc-maçons. Très proche de Saint Martin il s'était opposé à l'école rationaliste et s'était montré favorable à une réunion de toutes les théosophies sous une même mystique.

32 Lettres de l'Ohio., 110.

33 Ibid., 77.

34 André Beaunier, La jeunesse de Joseph Joubert (Paris, 1918), 283.

35 G. Chaussinand-Nogaret, La Noblesse au XVIIIe siècle (Paris, 1984), 15. 